\title{
Architectural and functional alterations of the small intestinal mucosa in classical Whipple's disease
}

\author{
H-J Epple ${ }^{1,3}$, J Friebel ${ }^{1,3}$, V Moos ${ }^{1,3}$, H Troeger $^{1}$, SM Krug ${ }^{2}$, K Allers ${ }^{1}$, K Schinnerling ${ }^{1,4}$, A Fromm ${ }^{2}$, \\ B Siegmund ${ }^{1}$, M Fromm ${ }^{2}$, JD Schulzke ${ }^{1,2,3}$ and T Schneider ${ }^{1,3}$
}

Classical Whipple's disease (CWD) affects the gastrointestinal tract and rather elicits regulatory than inflammatory immune reactions. Mechanisms of malabsorption, diarrhea, and systemic immune activation are unknown. We here analyzed mucosal architecture, barrier function, and immune activation as potential diarrheal trigger in specimens from 52 CWD patients. Our data demonstrate villus atrophy and crypt hyperplasia associated with epithelial apoptosis and reduced alkaline phosphatase expression in the duodenum of CWD patients. Electrophysiological and flux experiments revealed increased duodenal permeability to small solutes and macromolecules. Duodenal architecture and permeability ameliorated upon antibiotic treatment. Structural correlates for these alterations were concordant changes of membranous claudin-1, claudin-2, claudin-3, and tricellulin expression. Tumor necrosis factor- $\alpha$ and interleukin-13 were identified as probable mediators of epithelial apoptosis, and altered tight junction expression. Increased serum markers of microbial translocation and their decline following treatment corroborated the biological significance of the mucosal barrier defect. Hence, mucosal immune responses in CWD elicit barrier dysfunction. Diarrhea is caused by loss of absorptive capacity and leak flux of ions and water. Downregulation of tricellulin causes increased permeability to macromolecules and subsequent microbial translocation contributes to systemic inflammation. Thus, therapeutic strategies to reconstitute the mucosal barrier and control inflammation could assist symptomatic control of CWD.

\section{INTRODUCTION}

Classical Whipple's disease (CWD) is a chronic infection caused by Tropheryma whipplei. It primarily affects the small intestine but can also spread to other organ systems. Most patients with CWD suffer from gastrointestinal symptoms such as chronic diarrhea and malabsorption. Although these symptoms clearly indicate a dysfunction of the small intestinal mucosa, the underlying pathomechanisms have not been elucidated so far. Therefore, the question how the mucosal infection in CWD is linked to the gastrointestinal symptoms of the patients is unresolved.

Histologically, CWD is characterized by small intestinal accumulation of mucosal macrophages, which are densely packed with periodic acid-Schiff (PAS)-stained granules corresponding to intracellular bacteria. ${ }^{1}$ One would intuitively assume a link between the massive alterations of mucosal macrophages and the gastrointestinal symptoms of CWD patients. Obviously, mucosal inflammation, induced by chronic infection, causing gastrointestinal symptoms by inflammatory damage of the mucosal barrier, would be a likely candidate for such a link. However, up to now the small intestinal mucosal barrier and transport properties have not been assessed in CWD. Therefore, whether or not there is pathological impairment of the mucosal barrier and transport function in CWD was unknown. Furthermore, inflammatory reactions within the intestinal mucosa of CWD patients are dominated by regulatory rather than pathogen-directed immune mechanisms. ${ }^{2-9}$ On the other hand, CWD is characterized by systemic inflammation as reflected by elevated C-reactive protein, increased erythrocyte sedimentation rate, and leukocytosis. ${ }^{1,10}$

${ }^{1}$ Department of Gastroenterology, Rheumatology and Infectious Diseases, Charité-Universitätsmedizin Berlin, Campus Benjamin Franklin, Berlin, Germany and ${ }^{2}$ Institute of Clinical Physiology/Nutritional Medicine, Charité-Universitätsmedizin Berlin, Campus Benjamin Franklin, Berlin, Germany. Correspondence: V Moos (verena.moos@charite.de)

${ }^{3}$ These authors contributed equally to this work.

${ }^{4}$ Current address (after the end of the study): Facultad de Medicina, Programa Disciplinario de Inmunología, Universidad de Chile, Santiago, Chile.

Received 7 July 2016; accepted 10 January 2017; published online 8 February 2017. doi:10.1038/mi.2017.6 
Although the tolerogenic mucosal immune reaction was attributed to a subtle immune defect of the host and to antiinflammatory effects of $T$. whipplei, ${ }^{1,11}$ little is known about the causes of the systemic inflammation.

Taken together, the present knowledge about the pathogenesis of CWD is fragmentary. The mechanisms of diarrhea and malabsorption are largely unknown, as are the causes for the systemic chronic inflammation frequently encountered in this disease. It may seem surprising that the pathophysiology of a disease described more than 100 years ago has not been better elucidated so far. However, the lack of a specific animal model and the rarity of CWD are difficult preconditions for an indepth analysis of mucosal function.

To overcome these obstacles, we performed functional, structural, and immunological analyses in small intestinal mucosal samples obtained from 52 patients with CWD (17 before treatment, 30 both untreated and treated, and 5 only treated) over a prolonged period of time. We identified loss of absorptive capacity and leak flux of osmotically active solutes across a defective small intestinal mucosa as important causes for malabsorption and diarrhea. In addition, increased uptake of macromolecules from the gut lumen, including microbial components, owes to downregulation of the tight junction protein tricellulin and contributes to systemic inflammation in CWD. Successful treatment of CWD subsequently resulted in partial normalization of duodenal architecture, mucosal permeability, and surrogate markers of microbial translocation.

\section{RESULTS}

\section{Mucosal transformation in CWD}

Small intestinal absorption of nutrients and electrolytes occurs mainly in the surface epithelium (as opposed to the epithelium of the crypts). Therefore, the absorptive capacity of the small bowel is directly related to the exposed area of the surface epithelium, which under physiological conditions is greatly enhanced by villi and microvilli.

In our untreated CWD patients, we observed marked villus atrophy and moderate crypt hyperplasia, as indicated by a more than $50 \%$ reduction of villus length $(410 \pm 21 \mu \mathrm{m}$ in controls, $186 \pm 12 \mu \mathrm{m}$ in CWD; $P<0.0001$ ), and an $86 \%$ elongation of crypt length $(89 \pm 5 \mu \mathrm{m}$ in controls, $166 \pm 7 \mu \mathrm{m}$ in CWD; $P<0.0001$ ) (Figure 1a and $\mathbf{b}$ ). Upon antibiotic treatment and clinical remission of the disease, villus length was enhanced $(264 \pm 13 \mu \mathrm{m} ; P<0.001)$ and crypt length reduced $(136 \pm 6 \mu \mathrm{m}$; $P<0.001)$ compared to untreated patients, although normal values were not reached in this group of patients (Figure 1b).

Mucosal architecture depends on the balance between apoptosis of mature surface enterocytes and production of undifferentiated enterocytes within the crypts. ${ }^{12}$ Accordingly, we observed an increased number of epithelial apoptotic cells with clustering at the villus tips and increased numbers of Ki67-positive proliferating crypt cells in untreated as well as treated CWD patients (Figure 1a and c). Furthermore, visualization of epithelial alkaline phosphatase, which is a generally accepted marker of small intestinal mucosal differentiation, revealed a strong reduction in alkaline phosphatase expression in the surface epithelial cells in untreated CWD patients (Figure 1a).

Taken together, these data indicate a hyperregenerative mucosal transformation of the small intestinal mucosa in CWD with loss of differentiated surface epithelium, increased enterocyte apoptosis, and compensatory increase of crypt cell proliferation that ameliorate but do not completely normalize despite clinical remission.

\section{Small intestinal mucosal barrier function}

The single layer epithelium of the gut mucosa represents the most important physical barrier between the body interior and the gut lumen. Increased epithelial permeability to small molecules (molecular weight $(\mathrm{MW})<500 \mathrm{Da}$ ) causes a leak flux of osmotically active solutes (which are mainly sodium and chloride) and water into the lumen. Increased permeability to large molecules ( $\mathrm{MW}>1 \mathrm{kDa}$ ), on the other hand, would allow for increased uptake of microbial- and food-derived macromolecular components.

The permeability of the mucosal barrier to ions like sodium and chloride is electrophysiologically represented in a reciprocal manner by the ohmic resistance of the epithelial layer $\left(R^{\text {epi }}\right)$. Thus, the diffusion barrier of the mucosa to ions is determined by $R^{\mathrm{epi}}$, whereas subepithelial tissues (represented by $R^{\text {sub }}$ ) do not significantly contribute, because capillaries reach into the immediate vicinity of the epithelium. ${ }^{13}$ In order to quantify $R^{\mathrm{epi}}$, we performed impedance spectroscopy, which allows for differentiation between $R^{\text {epi }}$ and $R^{\text {sub }}$. Original impedance locus plots are depicted in Figure 2a. The duodenal $R^{\text {epi }}$ of untreated CWD patients amounted to only about $60 \%$ of controls $\left(17.3 \pm 1.3\right.$ vs. $\left.27.6 \pm 1.8 \Omega \mathrm{cm}^{2}, P<0.001\right)$ and normalized upon treatment $\left(25.4 \pm 2.4 \Omega \mathrm{cm}^{2}, \quad P<0.05\right.$ compared to untreated CWD). There was no significant difference in the duodenal $R^{\text {sub }}$ between untreated and treated CWD and controls (Figure 2b). Mannitol fluxes across the duodenal mucosa of untreated CWD patients were also increased over controls $\left(10.4 \pm 1.9\right.$ vs. $4.5 \pm 0.610^{-6} \mathrm{~cm} \mathrm{~s}^{-1}$, $P=0.019$; corrected for the quotient $\left.R^{\mathrm{t}} / R^{\mathrm{epi}}\right)$, but remained elevated despite clinical remission $\left(8.8 \pm 1.010^{-6} \mathrm{~cm} \mathrm{~s}^{-1}\right.$; corrected for the quotient $R^{\mathrm{t}} / R^{\mathrm{epi}}$ ) (Figure 2c). These results indicate a barrier defect to small ions and low molecular weight solutes in untreated CWD. The resolution of diarrhea in treated patients corresponds to a normalization of the epithelial sodium and chloride permeability confirming leak flux as a diarrheal mechanism in CWD.

\section{Macromolecular permeability and microbial translocation}

The permeability of the duodenal mucosa of CWD patients to large molecules was directly quantified by flux measurements with dialyzed, fluorescein isothiocyanate (FITC)-labeled dextran (FITC-labelled dextran 4000 (FD4), MW 4kDa) and with horseradish peroxidase (HRP, MW $44 \mathrm{kDa}$ ). As shown in Figure 3a, in untreated CWD the permeability to FD4 was five-fold increased over controls $(9.0 \pm 2.2$ vs. $1.8 \pm 0.7$ $\left.10^{-7} \mathrm{~cm} \mathrm{~s}^{-1}, P=0.004\right)$ and that to HRP 8.6-fold (9.2 \pm 2.5 vs. 

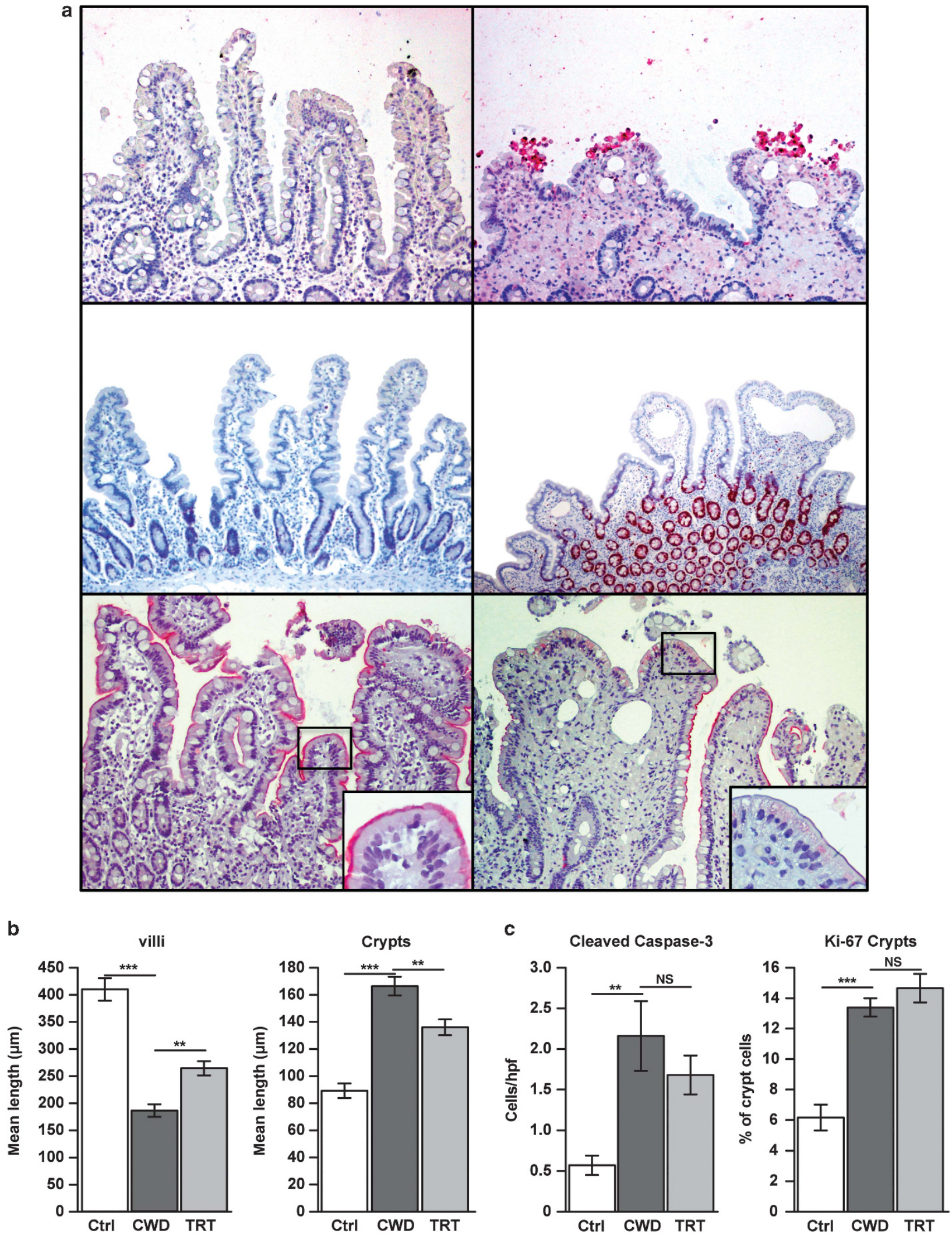

Figure 1 Transformation of the small intestinal mucosa in patients with classical Whipple's disease (CWD). (a) Representative sections of duodenal mucosa obtained from patients with CWD (right panel) or healthy controls (left panel). Upper panel: apoptotic cells visualized by caspase-3 staining (red). Middle panel: proliferating cells visualized by Ki-67 staining (red). Lower panel: mucosal alkaline phosphatase expression (red). Magnification $\times 200$. (b) Quantification of mucosal morphometric changes. (c) Quantification of epithelial cells positive for caspase-3 or Ki-67. ${ }^{* \star} P<0.01$, ${ }^{* \star *} P<0.001 . N=19-28$ for controls (Ctrl), untreated CWD (CWD), and treated CWD (TRT) patients, respectively. 


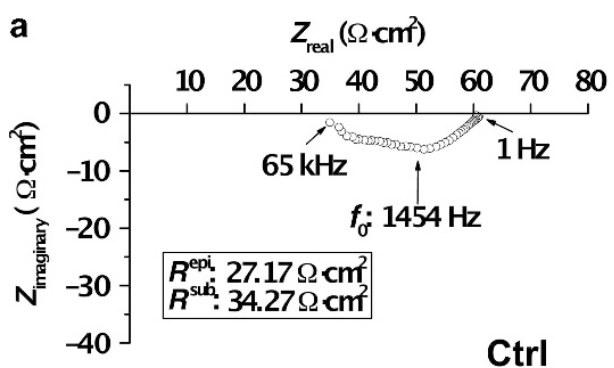

b

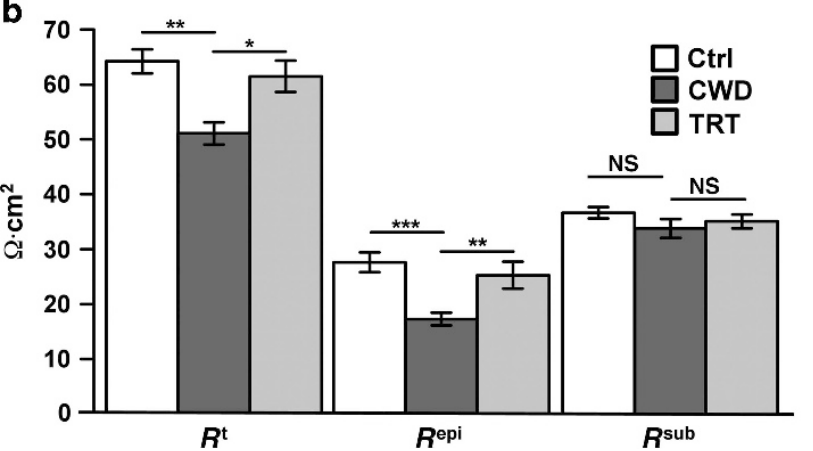

$Z_{\text {real }}\left(\Omega \cdot \mathrm{cm}^{2}\right)$

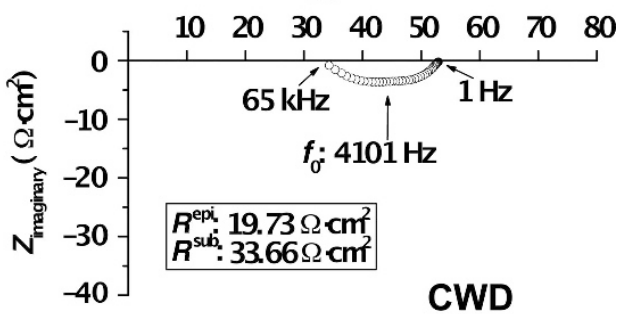

c

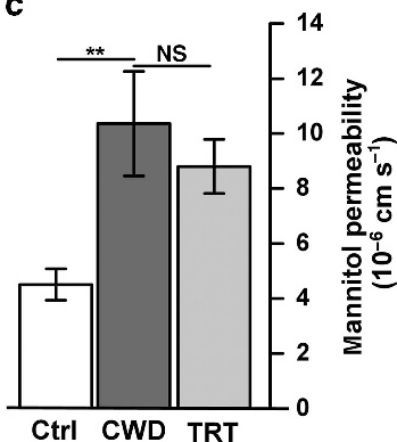

Figure 2 Small intestinal permeability to small molecules in patients with classical Whipple's disease (CWD). (a) Representative impedance locus plots of duodenal specimens obtained from a patient with CWD and a healthy control. (a) $Z_{\text {real }}$ gives the Ohmic component and $Z_{\text {imaginary }}$ the reactive component of the complex impedance constituted by the capacitive properties of the epithelium. The epithelial resistance equals the amount of $Z_{\text {real }}$ between the intersections of the $Z_{\text {imaginary }}$ vs. $Z_{\text {real }}$ plots with the $x$ axis. (b) Quantification of $R^{\mathrm{epi}}, R^{\text {sub }}$, and $R^{t}$ from $N=7$ controls (Ctrl), 7 untreated CWD (CWD) patients, and 5 treated CWD (TRT) patients. (c) Paracellular permeability $\left(P_{\text {mannitol }}\right)$ was determined from mucosal-to-serosal ${ }^{3} \mathrm{H}$-mannitol fluxes at a mannitol concentration of $10 \mathrm{~mm}$ corrected for the individual quotient $R^{\mathrm{t}} / R^{\mathrm{epi}}$ from $N=7$ controls, $6 \mathrm{CWD}$ patients, and 5 TRT patients. ${ }^{\star} P<0.05, P<0.01$, ${ }^{* \star *} P<0.001$

$\left.1.1 \pm 0.410^{-8} \mathrm{~cm} \mathrm{~s}^{-1}, P=0.029\right)$. Both parameters normalized completely upon treatment. In line with these results, lipopolysaccharides (LPS) were increased in the serum of untreated CWD patients as well as lipopolysaccharide-binding protein (LBP) and soluble CD14 (sCD14), indicating increased microbial translocation from the gut lumen (Figure $3 \mathbf{b}$ ). LPS values remained elevated, LBP and SCD14 normalized following treatment (Figure $\mathbf{3 b}$ ). Taken together, these results provide both direct and indirect evidence for increased microbial translocation owing to a pathological mucosal permeability to large molecules in untreated CWD that improves in treated patients.

\section{Active ion secretion of the small intestinal mucosa}

Although we addressed passive solute secretion by quantification of mucosal permeability to small molecules as outlined above, active ion secretion was investigated by additional experiments. In the small intestine, active ion secretion is mainly based on chloride and bicarbonate. Because these are electrogenic in nature, they can be quantified by short circuit current (Isc) measurements with or without pharmacological stimulation and inhibition with bumetanide.

In a series of electrophysiological experiments $(n=6$ for each data set), we did not find any differences between untreated CWD patients and controls in (i) the spontaneous occurring "basal" Isc, which was determined after equilibrium of the epithelia in the Ussing chamber $(87.6 \pm 13.0$ vs. $95.4 \pm 10.9 \mu \mathrm{A}$ $\mathrm{cm}^{-2}, P=0.65$ ), (ii) the Isc induced by stimulation with theophylline and $\mathrm{PEG}_{2}\left(55.3 \pm 24.3\right.$ vs. $69.7 \pm 13.3 \mu \mathrm{A} \mathrm{cm}{ }^{-2}$, $P=0.63$ ), and (iii) the bumetanide-sensitive Isc after theophylline/PEG 2 stimulation $\left(26.9 \pm 8.5\right.$ vs. $29.0 \pm 10.1 \mu \mathrm{A} \mathrm{cm}^{-2}$, $P=0.87$ ). Thus, there was no evidence for pathologically increased active small intestinal ion secretion in CWD.

\section{Tight junction proteins associated with increased permeability to small and large molecules}

Function and integrity of the epithelial barrier of the intestinal mucosa is mediated and maintained by a meshwork of tight junction strands formed by proteins, which connect adjacent enterocytes. Tight junction proteins are considered to be central regulators of paracellular barrier function, and changes of the abundance of certain tight junction proteins have been shown to be associated with corresponding changes of epithelial permeability. ${ }^{14,15}$

We analyzed the expression of a panel of tight junction proteins known to be expressed in the small bowel ${ }^{15}$ by immunoblotting (Figure 4a) and subsequent densitometry (Figure $4 \mathrm{~b}$ ). For the purpose of our study, tight junction proteins can be subdivided into three groups: "barrier formers" (causing decreased paracellular permeability to all solutes; e.g., claudins 1, 3, 4, 5, 7), "paracellular channel formers" (causing increased paracellular permeability to ions, small solutes, and water; e.g., claudins 2, 15), and "macromolecule barrier formers" (causing increased paracellular permeability to large molecules; occludin, tricellulin). ${ }^{14,16}$ The intestinal mucosa of patients with untreated CWD showed altered expression of 

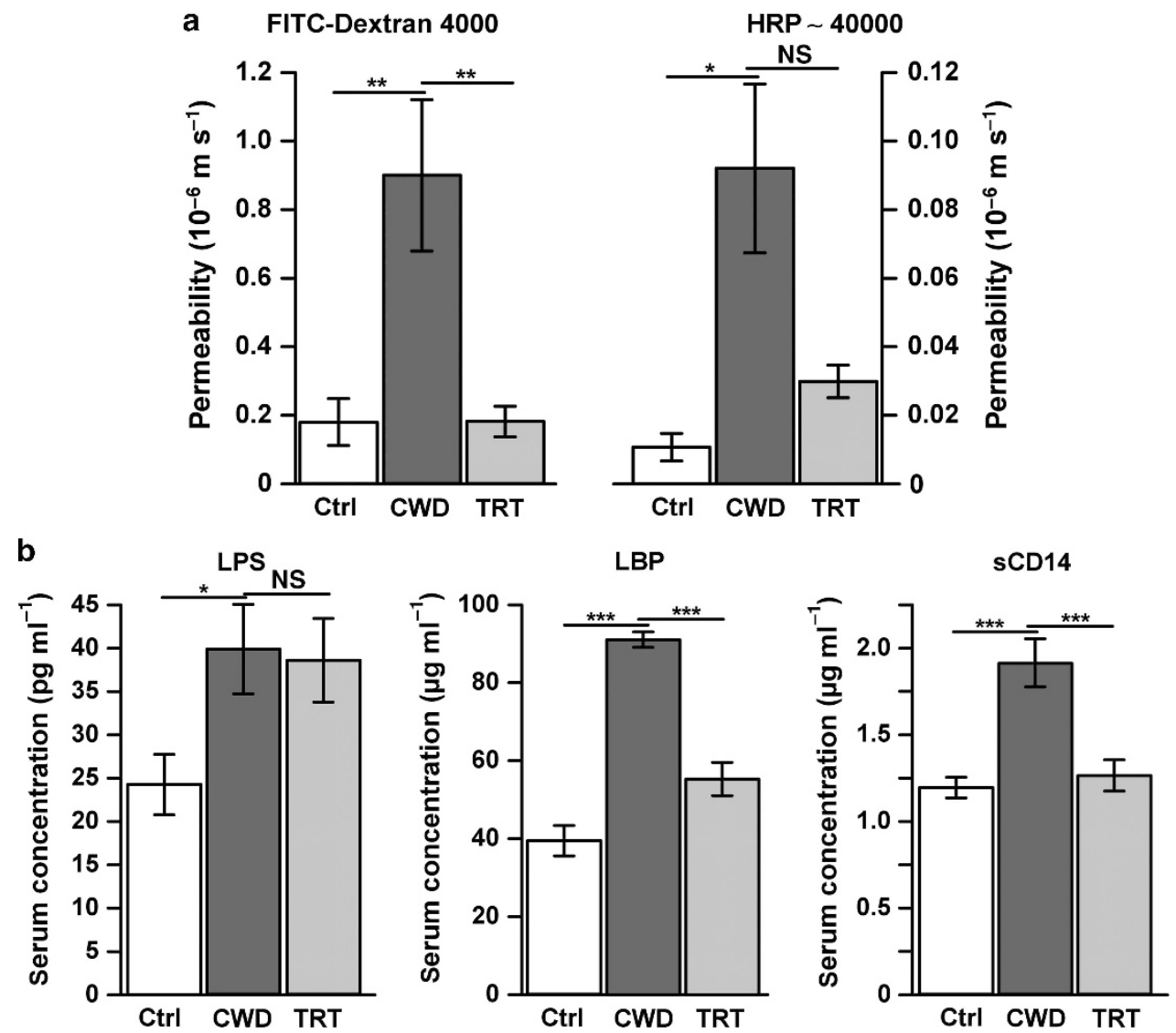

Figure 3 Small intestinal translocation of macromolecules in patients with classical Whipple's disease (CWD). (a) Mucosal permeability to FITCdextran (FD4, molecular weight (MW) $4 \mathrm{kDa}$ ) and horseradish peroxidase (HRP, MW 40 kDa) determined by flux studies as described in the Methods section. $N=7$ (HRP, FD4) for controls (Ctrl), $N=5$ (HRP) and 4 (FD4) for untreated CWD (CWD) patients, and $N=4$ (HRP) and 5 (FD4) for treated CWD (TRT) patients, respectively. (b) Serum concentration of markers for microbial translocation: lipopolysaccharides (LPS), lipopolysaccharide-binding protein (LBP), and soluble CD14 (sCD14). $N=22$ for Ctrl, and $N=20$ for CWD and TRT patients, respectively. ${ }^{\star} P<0.05,{ }^{\star \star} P<0.01,{ }^{\star \star \star} P<0.001$.

proteins of all three kinds. The expression of the "barrier formers" claudin- 1 and claudin-3 was reduced to $35 \%$ and $58 \%$ of controls, respectively, that of the "paracellular channel former" claudin-2 was increased to more than $200 \%$ of controls, and the expression of the "macromolecule barrier former" tricellulin was markedly suppressed to $17 \%$ of controls (Figure $\mathbf{4 b}$ ). The observed changes in tight junction protein expression are fully consistent with our functional analysis. Both increased enterocyte apoptosis and altered protein composition of the epithelial tight junctions can be regarded as the structural correlates of the increased small intestinal permeability to small and large molecules in untreated CWD.

\section{Mediators of mucosal alterations in CWD}

Having identified epithelial apoptosis and altered tight junction protein expression as important structural changes within the small intestinal mucosa of untreated CWD patients, we tried to gain some insight into the mediating mechanisms involved. In various intestinal inflammatory or infectious disorders mucosal barrier dysfunction has been linked to the action of mucosal cytotoxic T lymphocytes ${ }^{17-19}$ and/or mucosal cytokines. Immunologically, CWD is characterized by altered macrophage function, markedly reduced T-helper-1 responses and a shift of cytokine production toward T-helper-2 (Th2) cell activity. $^{2,59}$ Therefore, we focused our analysis of mucosal production on macrophage-derived (tumor necrosis factor- $\alpha$ $(\mathrm{TNF}-\alpha)$ ) and Th2 (interleukin-4 (IL-4), IL-13) cytokines, which have been previously shown to be important regulators of the mucosal barrier, acting via apoptosis induction or by perturbation of epithelial tight junction expression. ${ }^{19-21}$

As shown in Figure 5a and $\mathbf{b}$, the mucosal density of perforin-positive mucosal $\mathrm{T}$ cells in untreated CWD patients was not different from that of controls. On the other hand, the mucosal production of TNF- $\alpha$, IL-13, and IL- 4 was markedly increased in CWD patients (Figure $\mathbf{5 c}$, $\mathbf{e}$ and $\mathbf{f}$ ). Furthermore, mucosal production of the macrophage activation marker sCD14 was also increased in samples obtained from CWD patients (Figure 5d). Thus, the mucosal barrier defect in CWD is probably mediated by mucosal cytokines secreted by mucosal macrophages and Th2-polarized CD4 T cells. The concerted action of these cytokines cause epithelial apoptosis and changes of tight junction protein expression. Epithelial apoptosis leads to villus atrophy, and both epithelial apoptosis and altered tight junctions cause impairment of the mucosal barrier function. 
a
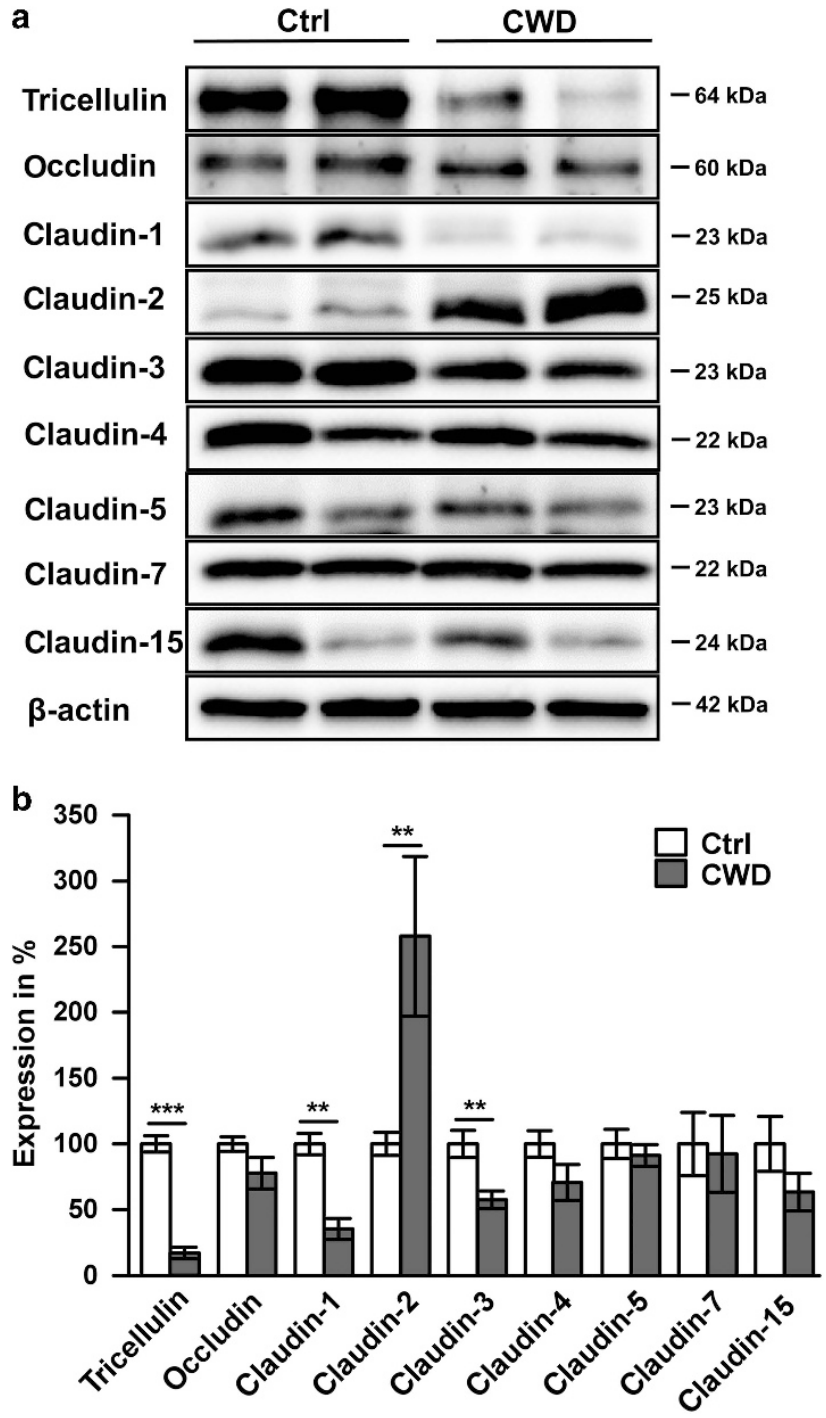

Figure 4 Quantitative analysis of membrane-bound tight junction proteins in untreated classical Whipple's disease (CWD). Tight junction protein expression was quantified by immunoblotting with subsequent densitometric analysis. (a) Representative immunoblots. (b) Densitometric quantification. Values represent protein expression in the small intestinal mucosa of patients with CWD in relation to the respective protein expression of healthy controls (set as 100\%) determined on the same blot with $N=6-8$ for each protein obtained from untreated CWD (CWD) patients or controls (Ctrl), respectively. ${ }^{* *} P<0.01,{ }^{* * *} P<0.001$.

\section{DISCUSSION}

CWD is a chronic infectious disease characterized by massive accumulation of T. whipplei in macrophages located within the small intestinal mucosa. During the last decade great progress has been made toward a better understanding of the immunological features predisposing a susceptible host to CWD. ${ }^{2,3,5,9,11}$ However, what causes the major gastrointestinal and systemic clinical symptoms of the disease-chronic diarrhea, malabsorption, and systemic inflammation-is largely unknown. In the present study, we performed detailed morphological, functional, and immunological analyses on a

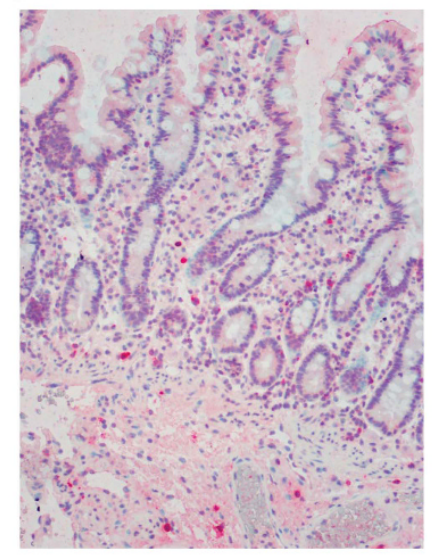

b

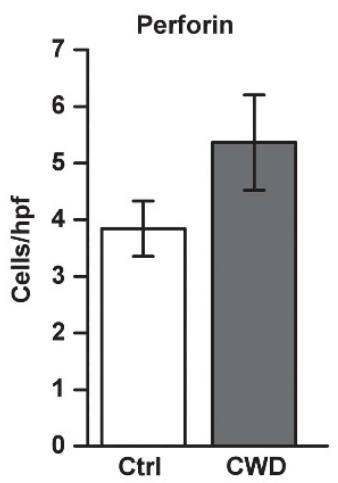

c
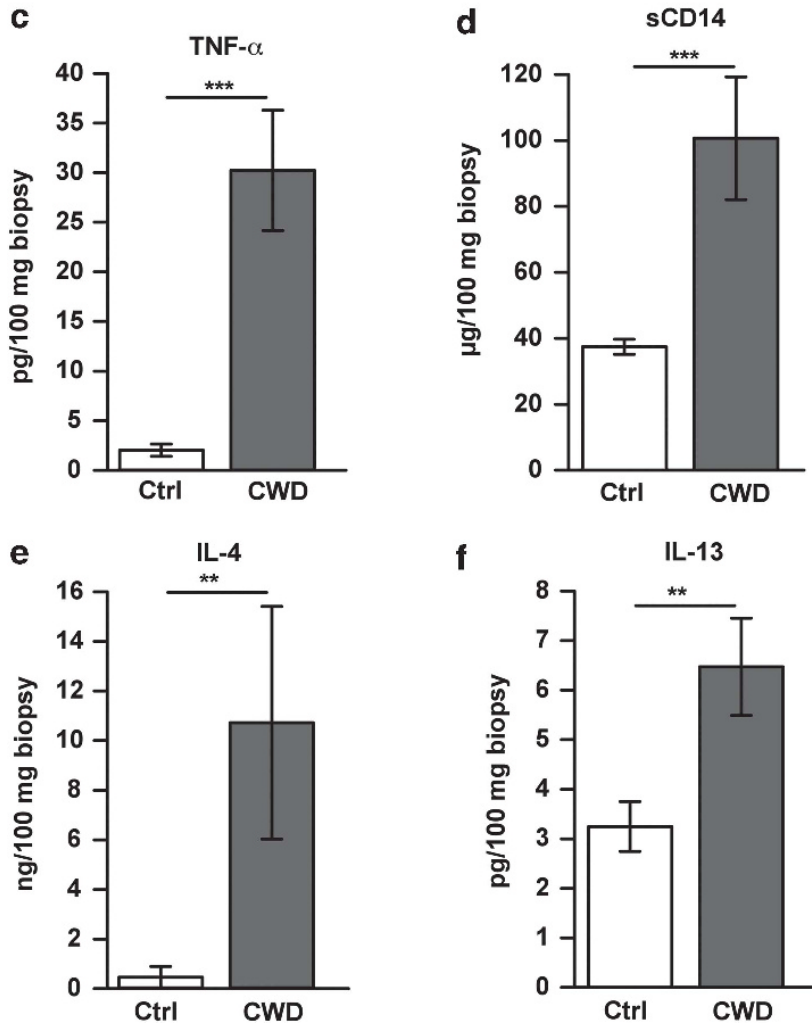

$\mathbf{f}$

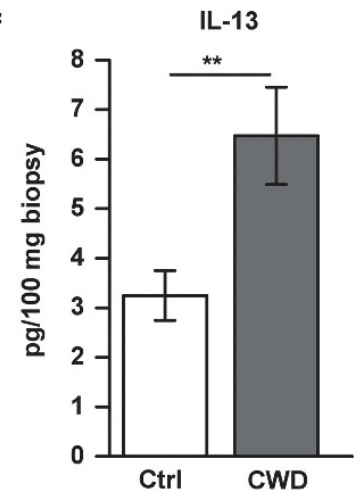

Figure 5 Mucosal cytotoxic T cells and mucosal cytokine production. (a) Representative immunohistochemical analysis and (b) quantification of mucosal cytotoxic $\mathrm{T}$ cell as determined by perforin-positive mononuclear cells. $N=31$ for controls (Ctrl) and $N=19$ for untreated classical Whipple's disease (CWD) (CWD) patients, respectively.

(c) Mucosal production of tumor necrosis factor alpha (TNF- $\alpha$ ) and

(d) soluble CD14 (sCD14). N=10 and 15 (sCD14, TNF- $\alpha$ ) for controls, and $N=6$ and 5 for CWD patients, respectively. (e) Mucosal production of T-helper-2 (Th2) cytokines interleukin-4 (IL-4) and (f) IL-13. $N=15$ and 18 (IL-4, IL-13) for controls, and $N=5$ and 10 for CWD patients, respectively. ${ }^{\star \star} P<0.01,{ }^{\star \star \star} P<0.001$.

mucosal samples obtained from patients with untreated and treated CWD. Our data indicate that the small intestinal mucosa in CWD is characterized by a significant barrier defect elicited by mucosal cytokines and linked to the systemic immune activation characteristic for CWD. 
The morphological analysis of the small intestinal samples obtained from CWD patients revealed a mucosal transformation in CWD similar to that of celiac disease. Thus, we found villus atrophy and crypt hyperplasia in the small intestinal mucosa of untreated CWD patients, and like in celiac disease, the alterations of mucosal architecture were linked to incomplete maturation and increased apoptosis of surface epithelial cells. ${ }^{22,23}$ The functional consequences of alterations of mucosal architecture reflect the spatial organization of small intestinal function. Thus, small intestinal absorption mainly occurs in villus epithelium and depends on the apical enzyme activity of terminally differentiated surface epithelial cells. Villus atrophy and dysmaturation of surface epithelial cells, as observed in CWD, therefore imply significant loss of absorptive capacity of the small intestine and thus contribute to malabsorption and diarrhea in diseased patients. The partial recovery of duodenal architecture upon antibiotic treatment seems to be sufficient to achieve clinical remission of the patients.

Our functional analysis not only targeted absorptive but also secretory properties of the small intestinal mucosa in CWD. Whereas active ion secretion was not stimulated, we observed increased permeabilities to sodium, chloride, and mannitol in the mucosa of CWD patients. Promoting passive efflux of sodium, chloride, and water, increased epithelial permeability to small molecules ( $\mathrm{MW}<500 \mathrm{Da})$ and ions has long been recognized as a diarrheal mechanism in its own right, and the term leak flux diarrhea has been coined to denote this condition. ${ }^{24-26}$ Thus, like in Crohn's disease, ulcerative colitis, celiac disease, and HIV enteropathy, ${ }^{27-29}$ diarrhea in CWD is caused by both decreased absorption and by increased leak flux of solutes and water into the gut lumen. Subsequent treatment of CWD enables complete recovery of the epithelial resistance and resolves leak flux. On the other hand, the persistently increased mannitol permeability in treated patients indicates a subtle persistent defect of the integrity of the mucosal barrier that is not sufficient to quantitatively increase sodium and water efflux, and to elicit diarrhea.

In the absence of ongoing active transepithelial transport, ions cannot easily permeate the epithelium via the transcellular route. Therefore, increased epithelial ion permeability mainly reflects respective changes of paracellular permeability. The paracellular pathway in turn has been shown to be composed of two paracellular diffusion routes distinguished from each other by size-selectivity and regulation. The "restrictive pathway" has a high capacity for passage of small ions and uncharged solutes but does not permit translocation of macromolecules. Its permeability properties are mainly determined by the claudin composition of the epithelial tight junctions. ${ }^{14,30}$ Accordingly, in untreated CWD samples there was a clear reduction of the barrier-forming proteins claudin-1 and -3. The expression of the paracellular channel-forming protein claudin-2, on the other hand, was more than two-fold increased. Regarding the restrictive pathway, CWD shares many features with other small intestinal enteropathies associated with diarrhea, such as Crohn's disease, celiac disease, and HIV enteropathy. In all these conditions (if untreated), the epithelial resistance was reduced compared to healthy controls and the expression of claudin-2 was increased. ${ }^{27-29}$

In contrast to the restrictive pathway, the non-restrictive paracellular pathway is permissive to passage of macromolecules but, owing to its low capacity, does not quantitatively contribute to ion efflux and diarrhea. ${ }^{14,30}$ In our untreated CWD patients, we demonstrated a pathological permeability of the non-restrictive pathway in CWD by increased mucosal permeability to the paracellular marker FITC-dextran- 4000 (MW $4 \mathrm{KDa}$ ) that normalizes completely upon antibiotic treatment. The molecular basis of mucosal macromolecule permeability has long been unknown. Channels formed by claudins are considered much too small to account for macromolecule translocation. Only during the recent years, occludin and tricellulin have been identified as main regulators of the non-restrictive pathway. ${ }^{14,16,31}$ In our CWD patients occludin expression was unaltered, but we found a strong reduction of epithelial tricellulin. Tricellulin is a tight junction protein first described in $2005 .{ }^{32}$ Like occludin, it belongs to the group of MARVEL domain proteins (MAL and related proteins for vesicle trafficking and membrane link). ${ }^{33}$ Unlike occludin and the claudins, tricellulin is not uniformly distributed along the tight junctions, but predominantly located at contact sites of three adjacent enterocytes (the tricellular junction). ${ }^{32}$ Recent evidence revealed a unique function of tricellulin for the epithelial permeability to macromolecules. Knockdown of tricellulin increased mucosal permeability for FITC-dextran4000 and, vice versa, was reduced by tricellulin overexpression. ${ }^{31,32}$ On the basis of these and additional supportive molecular and functional data obtained in various epithelial cell culture models, it is presently assumed that tricellulin expression is inversely linked to mucosal permeability to macromolecules. ${ }^{14,31,32}$ Our data are in excellent agreement with this conception. Thus, they corroborate the biological significance of tricellulin as a negative regulator of mucosal macromolecule permeability in a well-defined small intestinal disease. Accordingly, the pathological increase of macromolecule permeability in CWD can, at least partly, be attributed to reduced small intestinal tricellulin expression.

Differing from the paracellular route traveled by polysaccharide macromolecules, the translocation of protein macromolecules such as HRP (MW $44 \mathrm{kDa}$ ) seems to occur largely via transcytosis. ${ }^{34}$ Increased endocytotic uptake of HRP has been described in celiac disease ${ }^{34}$ and in inflamed tissue of Crohn's disease. ${ }^{35,36}$ Thus, the enhanced permeability to the tracer HRP in untreated CWD patients indicated increased transcytosis of protein macromolecules, which may also contribute to translocation of bacterial components.

As outlined above, a similar pattern of claudin dysregulation with upregulation of claudin-2 and downregulation of sealing claudins has been found in various chronic infectious or inflammatory diseases of the intestinal mucosa such as HIV infection, celiac disease, or inflammatory bowel disease. ${ }^{15,29}$ Consistent with our data, claudin dysregulation in these conditions is thought to be elicited by cytokines such as TNF- $\alpha$ 
and IL-13. ${ }^{15}$ Both, TNF- $\alpha$ and IL-13 are known to be potent inducers of epithelial apoptosis, and TNF- $\alpha$ enhances intestinal metalloproteinase expression..$^{20,29,37-39}$ Therefore, these cytokines also seem to drive villus atrophy in CWD. Although messenger RNA for TNF- $\alpha$ seems not enhanced in the duodenal mucosa in $\mathrm{CWD},{ }^{2}$ its protein expression by intestinal macrophages might be enhanced through increased translation induced by LPS, ${ }^{40}$ whereas the major source of IL-13 in CWD needs to be identified.

Taken together, the mucosal barrier defect in CWD is probably triggered by macrophage-derived TNF- $\alpha$ and the Th2 cytokine IL-13, and thus closely linked to the immunopathogenesis of the disease. ${ }^{11}$ Previous studies demonstrated a preponderance of immunoregulatory responses in CWD patients as reflected by a reduction of numerical and functional reduction of classically activated macrophages in favor for enhanced presence of alternatively activated M2 macrophages in the peripheral blood and the duodenal mucosa. ${ }^{2,3}$ In addition, flow cytometry and functional assays demonstrated that inflammatory reactions against $T$. whipplei are prevented by impaired maturation and IL-12 production of dendritic cells, an increased frequency and activation of functionally active regulatory $\mathrm{T}$ cells, a higher sensitivity of $\mathrm{T}$ cells to regulation, a predominance of Th2 activity accompanied by a reduced unspecific T-helper-1 reactivity, and, most importantly, the absence of T. whipplei-specific T-cell reactions in the blood and duodenal lamina propria. ${ }^{2-6,9}$ These cellular deficiencies are reflected by low serum concentrations of $\mathrm{IgG}_{2}$ and of T. whipplei-specific immunoglobulins in CWD patients and reduced duodenal secretion of $\operatorname{IgA}$ and $\operatorname{IgG}_{2}$ with only low amounts of T. whipplei-specific IgA, ${ }^{6-8}$ The predominance of tolerogenic reactions was interpreted as a permissive factor allowing for the persistence and spread of T. whipplei. ${ }^{1,5,9,11} \mathrm{In}$ this context, symptoms and signs of systemic inflammation such as fever, elevated blood sedimentation rate, and C-reactive protein were difficult to explain because the evidence outlined above and the usual absence of T. whipplei from the blood clearly argues against a pathogen-directed immune response as their cause. On the other hand, microbial translocation is regarded a well-established mechanism of systemic inflammation. ${ }^{41}$

Our data provide for the first time a logical coherence by linking a barrier defect of the intestinal mucosa to systemic immune activation of CWD patients. According to these findings, increased translocation of gut microbial components across a defective mucosal barrier is an important stimulator of systemic inflammation in CWD. In fact, even smallest amounts of LPS have been shown to elevate body temperature and to enhance serum concentration of inflammatory cytokines like TNF- $\alpha$ in healthy volunteers. ${ }^{42}$ In addition, LPS is able to directly stimulate CRP expression of human peripheral blood mononuclear cell. ${ }^{43}$ Thus, microbial translocation and systemic spread of microbial components might be directly responsible for systemic elevation of acute phase proteins despite a tolerogenic T-cell response and the lack of inflammatory macrophage functions in the gut. Upon improvement of the intestinal barrier in treated CWD, the normalization of LBP and $\mathrm{SCD} 14$ hints at a reduction of microbial translocation. Of the translocation markers analyzed, only LPS remains high in treated patients. However, the quantification of free LPS only reflects a small proportion of total LPS in the system, because most of the circulating LPS is bound and neutralized by a number of molecules, among them are LBP and cCD14. With the reduction of these molecules, free LPS cannot be cleared effectively anymore and thus remain elevated despite a reduction of microbial translocation from the gut.

In summary, we demonstrate that CWD is characterized by increased epithelial apoptosis, villus atrophy, and a dysfunctional barrier of the small intestinal mucosa. Like in Crohn's disease, ulcerative colitis, celiac diseases, and HIV enteropathy, ${ }^{15,29}$ loss of absorptive capacity and impairment of barrier function to small molecules are the underlying pathomechanisms of malabsorption and diarrhea in CWD. The mucosal barrier defect extends to a pathological permeability to macromolecules linked to downregulation of tricellulin. Therefore, systemic inflammation in CWD is probably partially driven by increased translocation of gut-derived microbial macromolecules. Upon treatment, epithelial resistance and macromolecular permeability return to normal, whereas persistent alterations in duodenal architecture, barrier function, and systemic LPS concentration indicate sustained abnormalities in CWD patients independent form active T. whipplei infection, which may contribute to the persistent symptoms found in these patients.

\section{METHODS}

Study subjects. All 52 CWD patients included (6 female, 46 male, mean age at diagnosis 55.9 years, range $23-73,17$ only untreated, 30 matched untreated and treated, 5 only treated; for details see Table 1; clinical specifications are summarized in Supplementary Table 1 online) exhibited gastrointestinal symptoms at time of diagnosis. In all patients, histological examination of duodenal biopsies revealed mucosal infiltration with PAS-positive macrophages. In addition, T. whipplei was detected in the duodenal biopsies of all patients by T. whipplei-specific PCR or by immunohistochemistry.

Duodenal mucosa specimens were obtained by forceps biopsy during routine upper gastrointestinal endoscopy. "Healthy" control subjects underwent endoscopy for cancer screening, unexplained anemia, or abdominal pain. Subjects were included as "healthy" controls, if signs of gastrointestinal or systemic inflammatory disease were absent. Exclusion criteria were inflammatory bowel disease, celiac disease, chronic hepatitis B or C, HIV infection, and any upper gastrointestinal tract pathology detected by endoscopy or histological examination. Duodenal biopsies were obtained from 57 control subjects (Table 1). Of these, biopsies from 20 patients were used for histological, and biopsies from 41 patients were used for electrophysiological, western blot, and cytokine analysis. Blood samples were collected from 22 patients. Table 1 provides numbers of patients and control subjects allocated to the analyzed specimens together with age and sex distribution.

Peripheral blood was collected in serum tubes (Vacutainer, BD Biosciences, Heidelberg, Germany) and either processed within $24 \mathrm{~h}$ or stored in polypropylene tubes at $-80^{\circ} \mathrm{C}$ until use. Biopsies were immediately processed to electrophysiological experiments or analysis of cytokine production or fixed in $4 \%$ paraformaldehyde (Sigma, Munich, Germany) and embedded in paraffin for immunohistochemical analysis. All subjects gave their written informed consent and 
Table 1 Specimens analyzed for the data presented

\begin{tabular}{|c|c|c|c|}
\hline & Untreated CWD patients & Treated CWD patients & Healthy control subjects \\
\hline Total & $\begin{array}{l}\text { Samples from } 47 \text { patients } \\
6 \mathrm{~F}, 41 \mathrm{M} ; 56.6 \text { y (42-73) }\end{array}$ & $\begin{array}{l}\text { Specimens from } 42 \text { samplings from } \\
35 \text { patients ( } 30 \text { patients matched } \\
\text { following treatment, } 5 \text { only treated) } \\
3 \mathrm{~F}, 32 \mathrm{M} ; 56.9 \text { y ( } 24-71) \\
21,5 \mathrm{~m} \text { (3-156) after diagnosis }\end{array}$ & $\begin{array}{l}\text { Samples from } 78 \text { subjects } \\
18 \mathrm{~F}, 60 \mathrm{M} ; 49.1 \text { y (16-85) }\end{array}$ \\
\hline \multicolumn{4}{|l|}{ Blood } \\
\hline Serum & $\begin{array}{l}\text { Samples from } 20 \text { patients } \\
2 \mathrm{~F}, 18 \mathrm{M} ; 55.7 \text { y (44-70) }\end{array}$ & $\begin{array}{l}\text { Samples from } 20 \text { patients } \\
2 \mathrm{~F}, 18 \mathrm{M} ; 57.2 \text { y }(45-71) \\
16.7 \mathrm{~m} \text { (3-35.5) after diagnosis }\end{array}$ & $\begin{array}{l}\text { Samples from } 22 \text { subjects } \\
7 \text { F, } 15 \text { M; } 54.6 \text { y (24-85) }\end{array}$ \\
\hline \multicolumn{4}{|l|}{ Duodenal biopsies } \\
\hline Total biopsies & $\begin{array}{l}\text { Samples from } 40 \text { patients } \\
5 \mathrm{~F}, 35 \mathrm{M} ; 54.4 \text { y (41-73) }\end{array}$ & $\begin{array}{l}\text { Samples from } 34 \text { patients } \\
3 \mathrm{~F}, 31 \mathrm{M} ; 57.9 \text { y }(24-71) \\
23.4 \mathrm{~m}(6-156) \text { after diagnosis }\end{array}$ & $\begin{array}{l}\text { Samples from } 57 \text { subjects } \\
11 \mathrm{~F}, 46 \mathrm{M} ; 46.8 \text { y (16-88) }\end{array}$ \\
\hline Biopsies for histology & $\begin{array}{l}\text { Samples from } 29 \text { patients } \\
3 \text { F, } 26 \mathrm{M} ; 58.4 \text { y (44-70) }\end{array}$ & $\begin{array}{l}\text { Samples from } 28 \text { patients } \\
3 \mathrm{~F}, 25 \mathrm{M} ; 58.1 \text { y }(45-71) \\
16.1 \mathrm{~m}(6-36) \text { after diagnosis }\end{array}$ & $\begin{array}{l}\text { Samples from } 20 \text { subjects } \\
2 \text { F, } 18 \text { M; } 54.4 \text { y (24-88) }\end{array}$ \\
\hline $\begin{array}{l}\text { Biopsies for electrophysiological, } \\
\text { cytokine, and western blot } \\
\text { analysis }\end{array}$ & $\begin{array}{l}\text { Samples from } 18 \text { patients } \\
2 \mathrm{~F}, 16 \mathrm{M} ; 54.1 \text { y }(41-70)\end{array}$ & $\begin{array}{l}\text { Samples from } 5 \text { patients }{ }^{a} \\
5 \mathrm{M} ; 57.2 \text { y (24-70) } \\
56.2 \mathrm{~m} \text { (6-156) after diagnosis }\end{array}$ & $\begin{array}{l}\text { Samples from } 41 \text { subjects } \\
9 \text { F, } 32 \text { M; } 42.8 \text { y (16-75) }\end{array}$ \\
\hline
\end{tabular}

CWD, classical Whipple's disease; F, female; M, male; m, months; y, years.

aOnly for electrophysiology.

the study was approved by the ethics committee of the Charite (registry numbers 229-17 and EA4/122/10).

Histology, immunohistochemistry, and morphometry. Immunostaining for apical membrane alkaline phosphatase, Ki-67, active caspase-3, and perforin was performed on paraffin sections of duodenal mucosa as described previously with rabbit anti-intestinal alkaline phosphatase (Abcam, Cambridge, UK), mouse anti-Ki-67 (DakoCytomation, Glostrup, Denmark), rabbit anti cleaved caspase-3 (Cell Signaling Technology, Boston, MA, USA), and rat IgG2a antiPerforin (Kamiya Biomedical, Seattle, WA, USA) using appropriate biotin-conjugated secondary antibodies, streptavidin-alkaline phosphatase, and Fast red as a substrate. ${ }^{2}$ Perforin-positive mononuclear cells were microscopically quantified in a blinded manner as cells per high power field (hpf; $0.237 \mathrm{~mm}^{2}$ ) and numbers from 10 to $20 \mathrm{hpf}$ were averaged. Ten villi and 30 contiguous crypt spaces were analyzed for caspase-3 or Ki-67-positive enterocytes. The crypt-villus-junction was defined as according to Holt et al. ${ }^{44}$ Mean villus height and crypt length was determined from five in a sagittal plane-orientated villi per subject using ImageJ software (NIH, Bethesda, MA, USA).

Electrophysiological experiments. Impedance spectroscopy was performed as described before. ${ }^{29}$ By application of alternating current of varying frequencies, this technique uses the capacitive properties of the epithelium to resolve the transmural wall resistance $\left(R^{t}\right)$ into its epithelial $\left(R^{\text {epi }}\right)$ and subepithelial $\left(R^{\text {sub }}\right)$ component. In order to quantify basal and stimulated electrogenic anion secretion, conventional short circuit current experiments were performed in Ussingtype chambers with pharmacologic stimulation or inhibition as indicated. All flux marker experiments were performed as mucosal-toserosal fluxes under voltage-clamp conditions and an exposed epithelial area of $0.049 \mathrm{~cm}^{2} .{ }^{3} \mathrm{H}$-mannitol fluxes were performed with $10 \mathrm{~mm}$ of unlabeled mannitol present on both sides and corrected for the quotient $R^{\mathrm{t}} / R^{\text {epi }} \cdot{ }^{29}$

For dextran flux measurements, $0.4 \mathrm{~mm}$ dialyzed FITC-labeled dextran-4000 (FD4, MW $4 \mathrm{kDa}$; TDB Consultancy AB, Uppsala, Sweden) and $0.4 \mathrm{~mm}$ unlabeled dextran-4000 (Sigma-Aldrich, Munich, Germany) was present on the apical and basolateral side, respectively. Three $30 \mathrm{~min}$ periods were analyzed for basolateral
FITC-dextran concentrations and FD4 fluxes were calculated as described previously. ${ }^{45}$ HRP fluxes were measured with $182 \mu \mathrm{M}$ (HRP, Sigma-Aldrich) on the apical side. Basolateral samples taken at 0, 30, $60,90,120 \mathrm{~min}$, and standards were analyzed using a fluorogenic peroxidase substrate (QuantaBlu, Pierce, Bonn, Germany) in a fluorometer (Tecan Infinite M200, Tecan, Männedorf, Switzerland) with $325 \mathrm{~nm}$ (excitation) and $420 \mathrm{~nm}$ (emission).

Immunoblots. For detection of tight junction protein expression, western blot analysis was performed on membrane extracts of duodenal biopsy specimens as described before. ${ }^{46}$ Primary antibodies were rabbit polyclonal IgG antibodies against claudin-1, -3, -5, -7, -15, occludin (Life Technologies GmbH, Darmstadt, Germany) and tricellulin (Sigma-Aldrich), and primary mouse monoclonal IgG antibodies against claudin-2, -4 (Life Technologies $\mathrm{GmbH}$ ) and $\beta$-actin (Sigma-Aldrich). Peroxidase-conjugated goat anti-rabbit IgG or goat anti-mouse IgG antibodies, and the chemiluminescence detection system Lumi-Light ${ }^{\text {PLUS }}$ western blotting substrate (SigmaAldrich) were used for detection of bound antibodies. Chemiluminescence signals were detected using a Fusion FX-7 imaging system (Vilber Lourmat, Eberhardzell, Germany) and densitometrically analyzed with the AIDA program package (Raytest, Berlin, Germany).

Duodenal cytokine secretion. Culture supernatants of duodenal biopsies were prepared as described elsewhere. ${ }^{47}$ Cytokine concentrations in supernatants of biopsy cultures were quantified by cytometric bead arrays (CBA, BD Biosciences) according to the manufacturer's protocols as previously described. ${ }^{3}$

Markers of microbial translocation. Levels of serum LPS and LBP, and sCD14 in serum and biopsy supernatants were analyzed by detection kits according to the manufacturers' recommendations. LPS was detected with the limulus amebocyte lysate chromogenic end-point assay (Charles River Laboratories, Wilmington, MA, USA) and levels of LBP and sCD14 were quantified by solid phase enzyme-linked immunosorbent assay (Hycult Biotech, Uden, the Netherlands).

Statistical analysis. Results are given as mean values \pm s.e.m. $\mathrm{N}$ indicates the number of specimens used for single, independent 
measurements. Statistical analysis was performed using Student's $t$-test for comparisons of healthy controls vs. untreated CWD, and untreated CWD vs. treated CWD with Bonferroni-Holm correction for multiple testing. $P<0.05$ was considered significant.

SUPPLEMENTARY MATERIAL is linked to the online version of the paper at http://www.nature.com/mi

\section{ACKNOWLEDGMENTS}

We thank Diana Bösel and Martina Seipel for excellent technical assistance, and Anika Geelhaar and Kristina Conrad for support in experimental setup. We are grateful to all patients, control subjects, and staff of the referring hospitals for help in obtaining samples.

This study was supported by the 5th Framework Program of the European Commission: QLG1-CT-2002-01049 and the Deutsche Forschungsgemeinschaft (KFO 104, SFB633, and SCHN 616/6-2, SCHU 559/ 11-3), and the Sonnenfeld-Stiftung Berli.

\section{AUTHOR CONTRIBUTIONS}

J.F., V.M. and H.-J.E., designed the study; J.F., V.M. and H.-J.E., K.S., K.A., A.F., S.M.K. and H.T. performed experiments; T.S., H.-J.E., J.D.S., H.T. and B.S. collected patient samples; J.F., V.M., H.-J.E., A.F. and K.S. analyzed data and made the figures; J.F., V.M., H.-J.E., K.A., J.D.S., T.S. and M.F. discussed and interpreted the data; V.M., T.S., M.F. and J.D.S. supervised the research; T.S., M.F., B.S. and J.D.S., applied for grants, H.-J.E., J.F. and V.M., wrote the manuscript, and all authors read and approved the final draft submitted.

\section{DISCLOSURE}

The authors declared no conflict of interest.

c) 2017 Society for Mucosal Immunology

\section{REFERENCES}

1. Schneider, T. et al. Whipple's disease: new aspects of pathogenesis and treatment. Lancet Infect. Dis. 8, 179-190 (2008).

2. Moos, V. et al. Impaired immune functions of monocytes and macrophages in Whipple's disease. Gastroenterology 138, 210-220 (2010).

3. Schinnerling, K. et al. Regulatory Tcells in patients with Whipple's disease. J. Immunol. 187, 4061-4067 (2011).

4. Schinnerling, K. et al. Role of dendritic cells in the pathogenesis of Whipple's disease. Infect. Immun. 83, 482-491 (2015).

5. Moos, V. et al. Reduced peripheral and mucosal tropheryma whippleispecific Th1 response in patients with Whipple's disease. J. Immunol. 177, 2015-2022 (2006).

6. Fenollar, F., Amphoux, B. \& Raoult, D. A paradoxical tropheryma whipplei western blot differentiates patients with whipple disease from asymptomatic carriers. Clin. Infect. Dis. 49, 717-723 (2009).

7. Marth, T. et al. Defects of monocyte interleukin 12 production and humoral immunity in Whipple's disease. Gastroenterology 113, 442-448 (1997).

8. Geelhaar, A. et al. Specific and nonspecific B-cell function in the small intestines of patients with Whipple's disease. Infect. Immun. 78, 4589-4592 (2010).

9. Marth, T. et al. Dysregulated peripheral and mucosal Th1/Th2 response in Whipple's disease. Gastroenterology 123, 1468-1477 (2002).

10. Feurle, G.E., Junga, N.S. \& Marth, T. Efficacy of ceftriaxone or meropenem as initial therapies in Whipple's disease. Gastroenterology 138, 478-486 (2010). quiz 11-2.

11. Marth, T. et al. Tropheryma whipplei infection and Whipple's disease. Lancet Infect. Dis. 16, e13-e22 (2016).

12. Shalimar, D.M. et al. Mechanism of villous atrophy in celiac disease: role of apoptosis and epithelial regeneration. Arch. Pathol. Lab. Med. 137, 1262-1269 (2013).

13. Tai, Y.H. \& Tai, C.Y. The conventional short-circuiting technique undershort-circuits most epithelia. J. Membr. Biol. 59, 173-177 (1981).

14. Krug, S.M., Schulzke, J.D. \& Fromm, M. Tight junction, selective permeability, and related diseases. Semin. Cell Dev. Biol. 36, 166-176 (2014).
15. Barmeyer, C., Schulzke, J.D. \& Fromm, M. Claudin-related intestinal diseases. Semin. Cell Dev. Biol. 42, 30-38 (2015).

16. Buschmann, M.M. et al. Occludin OCEL-domain interactions are required for maintenance and regulation of the tight junction barrier to macromolecular flux. Mol. Biol. Cell 24, 3056-3068 (2013).

17. Gitter, A.H. et al. Leaks in the epithelial barrier caused by spontaneous and TNF-alpha-induced single-cell apoptosis. FASEB J. 14, 1749-1753 (2000).

18. Di Sabatino, A. et al. Intraepithelial and lamina propria lymphocytes show distinct patterns of apoptosis whereas both populations are active in Fas based cytotoxicity in coeliac disease. Gut 49, 380-386 (2001).

19. Epple, H.J. et al. Acute HIV infection induces mucosal infiltration with CD4 + and CD8 + T cells, epithelial apoptosis, and a mucosal barrier defect. Gastroenterology 139, 1289-1300 (2010).

20. Heller, F. et al. Interleukin-13 is the key effector Th2 cytokine in ulcerative colitis that affects epithelial tight junctions, apoptosis, and cell restitution. Gastroenterology 129, 550-564 (2005).

21. John, L.J., Fromm, M. \& Schulzke, J.D. Epithelial barriers in intestinal inflammation. Antioxid. Redox Signal. 15, 1255-1270 (2011).

22. Moss, S.F. et al. Increased small intestinal apoptosis in coeliac disease. Gut 39, 811-817 (1996).

23. Maiuri, L. et al. FAS engagement drives apoptosis of enterocytes of coeliac patients. Gut 48, 418-424 (2001).

24. Fasano, A. et al. Vibrio cholerae produces a second enterotoxin, which affects intestinal tight junctions. Proc. Natl. Acad. Sci. USA 88, 5242-5246 (1991).

25. Moore, R. et al. C. difficile toxin A increases intestinal permeability and induces Cl- secretion. Am. J. Physiol. 259, G165-G172 (1990).

26. Stockmann, M. et al. Duodenal biopsies of HIV-infected patients with diarrhoea exhibit epithelial barrier defects but no active secretion. AIDS 12, 43-51 (1998)

27. Zeissig, S. et al. Changes in expression and distribution of claudin 2, 5 and 8 lead to discontinuous tight junctions and barrier dysfunction in active Crohn's disease. Gut 56, 61-72 (2007).

28. Schumann, M. et al. Defective tight junctions in refractory celiac disease. Ann. NY Acad. Sci. 1258, 43-51 (2012).

29. Epple, H.J. et al. Impairment of the intestinal barrier is evident in untreated but absent in suppressively treated HIV-infected patients. Gut 58, 220-227 (2009).

30. Günzel, D. \& Yu, A.S. Claudins and the modulation of tight junction permeability. Physiol. Rev. 93, 525-569 (2013).

31. Krug, S.M. et al. Tricellulin forms a barrier to macromolecules in tricellular tight junctions without affecting ion permeability. Mol. Biol. Cell 20, 3713-3724 (2009).

32. Ikenouchi, J. et al. Tricellulin constitutes a novel barrier at tricellular contacts of epithelial cells. J. Cell Biol. 171, 939-945 (2005).

33. Raleigh, D.R. et al. Tight junction-associated MARVEL proteins marveld3, tricellulin, and occludin have distinct but overlapping functions. Mol. Biol. Cell 21, 1200-1213 (2010).

34. Wheeler, E.E. et al. A morphological study of beta-lactoglobulin absorption by cultured explants of the human duodenal mucosa using immunocytochemical and cytochemical techniques. J. Pediatr. Gastroenterol. Nutr. 16, 157-164 (1993).

35. Malin, M. et al. Enhanced absorption of macromolecules. A secondary factor in Crohn's disease. Dig. Dis. Sci. 41, 1423-1428 (1996).

36. Söderholm, J.D. et al. Increased epithelial uptake of protein antigens in the ileum of Crohn's disease mediated by tumour necrosis factor alpha. Gut 53, 1817-1824 (2004).

37. Heller, F. et al. Epithelial apoptosis is a prominent feature of the epithelial barrier disturbance in intestinal inflammation: effect of pro-inflammatory interleukin-13 on epithelial cell function. Mucosal Immunol. 1 (Suppl 1), S58-S61 (2008).

38. Zeissig, S. et al. Downregulation of epithelial apoptosis and barrier repair in active Crohn's disease by tumour necrosis factor alpha antibody treatment. Gut 53, 1295-1302 (2004).

39. Di Sabatino, A. et al. Stromelysin-1 and macrophage metalloelastase expression in the intestinal mucosa of Crohn's disease patients treated with infliximab. Eur. J. Gastroenterol. Hepatol. 21, 1049-1055 (2009). 


\section{ARTICLES}

40. Han, J., Brown, T. \& Beutler, B. Endotoxin-responsive sequences control cachectin/tumor necrosis factor biosynthesis at the translational level. J. Exp. Med. 171, 465-475 (1990).

41. Mudd, J.C. \& Brenchley, J.M. Gut mucosal barrier dysfunction, microbial dysbiosis, and their role in HIV-1 disease progression. J. Infect. Dis. 214, S58-S66 (2016).

42. Dillingh, M.R. et al. Characterization of inflammation and immune cell modulation induced by low-dose LPS administration to healthy volunteers. J. Inflamm. 11 (2014).

43. Haider, D.G. etal. C-reactive protein is expressed and secreted by peripheral blood mononuclear cells. Clin. Exp. Immunol. 146, 533-539 (2006).
44. Holt, P.R., Pascal, R.R. \& Kotler, D.P. Effect of aging upon small intestinal structure in the Fischer rat. J. Gerontol. 39, 642-647 (1984).

45. Krug, S.M. et al. Sodium caprate as an enhancer of macromolecule permeation across tricellular tight junctions of intestinal cells. Biomaterials 34, 275-282 (2013).

46. Bürgel, N. et al. Mechanisms of diarrhea in collagenous colitis. Gastroenterology 123, 433-443 (2002).

47. Schneider, T. et al. Increased immunoglobulin G production by short term cultured duodenal biopsy samples from HIV infected patients. Gut 42 357-361 (1998). 